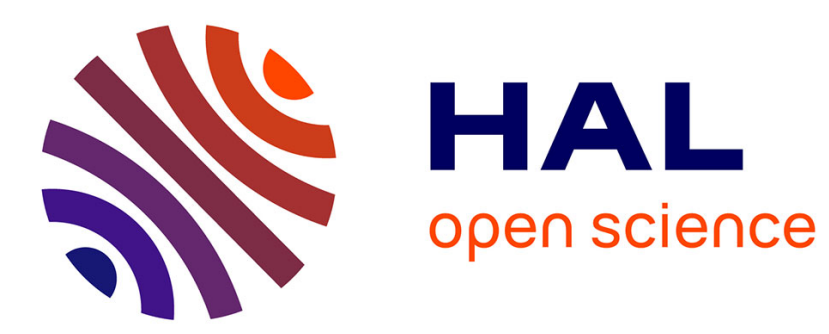

\title{
An effective model for the X 2A1-A 2B2 conical intersection in $\mathrm{NO} 2$
}

M. Joyeux, R. Jost, M. Lombardi

\section{To cite this version:}

M. Joyeux, R. Jost, M. Lombardi. An effective model for the X 2A1-A 2B2 conical intersection in NO2. Journal of Chemical Physics, 2003, 119, pp.5923-5932. 10.1063/1.1601602 . hal-00961311

\section{HAL Id: hal-00961311 https://hal.science/hal-00961311}

Submitted on 19 Mar 2014

HAL is a multi-disciplinary open access archive for the deposit and dissemination of scientific research documents, whether they are published or not. The documents may come from teaching and research institutions in France or abroad, or from public or private research centers.
L'archive ouverte pluridisciplinaire HAL, est destinée au dépôt et à la diffusion de documents scientifiques de niveau recherche, publiés ou non, émanant des établissements d'enseignement et de recherche français ou étrangers, des laboratoires publics ou privés. 


\title{
An effective model for the $X^{2} A_{1}-A^{2} B_{2}$ conical intersection in $\mathrm{NO}_{2}$
}

\author{
M. Joyeux, ${ }^{\text {a) }}$ R. Jost, and M. Lombardi \\ Laboratoire de Spectrométrie Physique (CNRS UMR 5588), Université Joseph Fourier-Grenoble 1, \\ BP 87, 38402 St. Martin d'Hères Cedex, France
}

(Received 14 May 2003; accepted 25 June 2003)

\begin{abstract}
We propose an efficient method for calculating the eigenstates and adjusting the parameters of an effective Hamiltonian, which reproduces the experimentally observed energy levels of $\mathrm{NO}_{2}$ up to $11800 \mathrm{~cm}^{-1}$ above the quantum mechanical ground state, that is a few thousands of $\mathrm{cm}^{-1}$ above the $X^{2} A_{1}-A^{2} B_{2}$ conical intersection, with a rms error less than $4 \mathrm{~cm}^{-1}$. This method principally relies on the determination, through first-order perturbation theory, of an optimal basis for each surface, which takes into account the nonresonant energy shifts experienced by the states of this surface. As a result, the size of the matrix, which one has to build and diagonalize to converge the spectrum up to $11800 \mathrm{~cm}^{-1}$, is of the order of 500-1000 instead of several tens of thousands. Thank to this Hamiltonian, the analysis of the experimental spectrum up to $11800 \mathrm{~cm}^{-1}$ could be completed. A detailed description of all states located above $9500 \mathrm{~cm}^{-1}$ is proposed, those lying below $9500 \mathrm{~cm}^{-1}$ being already known and tabulated. (C) 2003 American Institute of Physics.
\end{abstract}

[DOI: 10.1063/1.1601602]

\section{INTRODUCTION}

The Born-Oppenheimer separation of electronic and nuclear motions is a widely used approximation for interpreting molecular processes. Nonetheless, transitions between different electronic surfaces (non-Born-Oppenheimer, or nonadiabatic, dynamics) represent a field of growing interest in chemical physics, because they appear to govern a large variety of fundamental processes, such as internal conversion, intersystem crossing, electron transfer and photoinduced reactions. In this context, the conical intersection between the two lowest electronic surfaces of $\mathrm{NO}_{2}, X^{2} A_{1}$ and $A^{2} B_{2}$, has already attracted much attention from both the experimental (see Refs. 1-4, and references therein) and theoretical (see Refs. 5-13, and references therein) points of view. However, there still exists no model that satisfactorily reproduces the vibronic spectrum of $\mathrm{NO}_{2}$ in the region of the conical intersection, i.e., around $10000 \mathrm{~cm}^{-1}$. We recently made an attempt in this direction, by adjusting the parameters of a diabatic effective Hamiltonian against the energies of the lowest 283 vibronic states, which have now all been observed experimentally. ${ }^{14}$ The associated vibronic states, which extend up to $11400 \mathrm{~cm}^{-1}$ above the quantum mechanical ground state, consist of 276 states with predominant $X^{2} A_{1}$ ground electronic character and 7 states with predominant $A^{2} B_{2}$ excited electronic character. These calculations were based on the observation, that the net effect of the diabatic coupling between the two surfaces can be divided into two contributions, namely (i) a nonresonant coupling, which affects all of the $X^{2} A_{1}$ vibrational states but is associated to very weak mixing coefficients and (ii) a resonant coupling, which is significant (below $11400 \mathrm{~cm}^{-1}$ ) for only

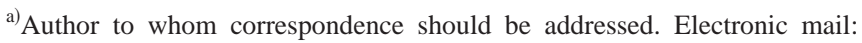
Marc.JOYEUX@ujf-grenoble.fr
}

few $X^{2} A_{1}$ states, but induces a significant mixing of these states with one (or few) energetically close $A^{2} B_{2}$ state $(\mathrm{s}) .^{15,16}$

The results obtained in this first work are perfectible in several ways. First, no effective Hamiltonian could be derived for the $A^{2} B_{2}$ excited electronic surface, because we did not calculate the energy shifts experienced by the states of this surface following the nonresonant couplings with the states of the ground electronic surface. Therefore, the excited electronic surface was rather described as a list of discrete energies. Moreover, the calculation procedure was rather approximate, since only very small matrices, corresponding to the resonantly coupled states of both electronic surfaces, were diagonalized. At last, the overlap matrix elements used in this first work were not precise enough, because of the loss of an unexpectedly large number of accuracy digits (several tens of them) following near cancellation of very large numbers.

The purpose of the present article is to report on the derivation of a complete effective Hamiltonian based on numerically exact quantum calculations. In this model, two Dunham expansions are used to describe, respectively, the uncoupled ground and excited diabatic electronic surfaces. The effective Hamiltonian for the ground electronic surface additionally includes a weak vibrational resonance. The third component of the effective Hamiltonian, i.e., the diabatic coupling surface, is taken as the usual $\lambda q_{3}$ interaction, where $q_{3}$ is the antisymmetric stretch normal coordinate for the electronic ground surface. When adjusting the parameters of such a model against experimental data, the main concern deals with CPU time, that is, in other terms, the size of the Hamiltonian matrix one has to build and diagonalize. Since $\lambda$ is of the order of several hundreds of $\mathrm{cm}^{-1}$, the diabatic coupling between states separated by several tens of thousands of $\mathrm{cm}^{-1}$ still has a sizable effect on the energy of each state. Moreover, each state is coupled to several tens of thou- 
sands of states belonging to the other surface and the resulting (nonresonant) energy shifts are cumulative. This explains why, when working with harmonic oscillator bases, the size of the Hamiltonian matrix, which is necessary to converge the spectrum up to 11000 or $12000 \mathrm{~cm}^{-1}$ above the ground state, is as large as several tens of thousands of vectors. These dimensions being not compatible with the large number of iterations required by an adjustment procedure, the work reported in this article principally relies on the choice of a different basis, which is obtained from first-order perturbation theory. It is however emphasized, that perturbation theory is used only to build the working basis, while the construction and diagonalization of the Hamiltonian matrix are numerically exact.

This point is explained in some detail in Sec. III. The effective Hamiltonian itself is described in Sec. II, while calculations and results are discussed in Sec. IV.

\section{THE EFFECTIVE HAMILTONIAN}

The effective diabatic Hamiltonian $\mathbf{H}$ is taken in the form,

$$
\mathbf{H}=\left(\begin{array}{ll}
H_{e} & H_{c} \\
H_{c} & H_{g}
\end{array}\right),
$$

where $H_{g}, H_{e}$, and $H_{c}$ describe the diabatic ground, excited and coupling surfaces, respectively. Let us note $\left(p_{1}, q_{1}\right)$, $\left(p_{2}, q_{2}\right)$, and $\left(p_{3}, q_{3}\right)$ the sets of conjugate coordinates for, respectively, the symmetric stretching, the bending, and the antisymmetric stretching modes of vibration around the minimum of the $X^{2} A_{1}$ ground electronic surface, and $\left|v_{1}, v_{2}, v_{3}\right\rangle$ the direct product basis for these three harmonic oscillators. In this basis, $H_{g}$ is taken as the sum of the eighth order Dunham expansion,

$$
\begin{aligned}
& \left\langle v_{1}, v_{2}, v_{3}\left|H_{g}\right| v_{1}, v_{2}, v_{3}\right\rangle \\
& =\sum_{i=1}^{3} \omega_{i} n_{i}+\sum_{i \leqslant j} x_{i j} n_{i} n_{j} \\
& \quad+\sum_{i \leqslant j \leqslant k} y_{i j k} n_{i} n_{j} n_{k}+\sum_{i \leqslant j \leqslant k \leqslant m} z_{i j k m} n_{i} n_{j} n_{k} n_{m},
\end{aligned}
$$

where $n_{i}=v_{i}+1 / 2(i=1,2,3)$, plus the $3 \omega_{1} \approx \omega_{2}+2 \omega_{3}$ vibrational resonance,

$$
\begin{aligned}
\left\langle v_{1}, v_{2}, v_{3}\left|H_{g}\right| v_{1}-3, v_{2}+1, v_{3}+2\right\rangle & \\
= & \left(k+\sum_{i=1}^{3} k_{i} n_{i}\right) \\
& \times \sqrt{v_{1}\left(v_{1}-1\right)\left(v_{1}-2\right)\left(v_{2}+1\right)\left(v_{3}+1\right)\left(v_{3}+2\right)},
\end{aligned}
$$

where $n_{1}=v_{1}-1, n_{2}=v_{2}+1$, and $n_{3}=v_{3}+3 / 2$. Let us similarly note $\left(p_{1}^{\prime}, q_{1}^{\prime}\right),\left(p_{2}^{\prime}, q_{2}^{\prime}\right)$, and $\left(p_{3}^{\prime}, q_{3}^{\prime}\right)$ the sets of conjugate coordinates for, respectively, the symmetric stretching, the bending and the antisymmetric stretching modes of vibration around the minimum of the $A^{2} B_{2}$ excited electronic surface, and $\left|v_{1}^{\prime}, v_{2}^{\prime}, v_{3}^{\prime}\right\rangle$ the direct product basis for these three harmonic oscillators. The experimental data for the ex- cited surface being much sparser than for the ground one, $H_{e}$ is taken to be just the energy difference between the bottoms of the two electronic surfaces plus a fourth order Dunham expansion,

$$
\begin{aligned}
& \left\langle v_{1}^{\prime}, v_{2}^{\prime}, v_{3}^{\prime}\left|H_{e}\right| v_{1}^{\prime}, v_{2}^{\prime}, v_{3}^{\prime}\right\rangle \\
& =E_{0}^{\prime}+\sum_{i=1}^{3} \omega_{i}^{\prime} n_{i}^{\prime}+\sum_{i \leqslant j} x_{i j}^{\prime} n_{i}^{\prime} n_{j}^{\prime},
\end{aligned}
$$

where $n_{i}^{\prime}=v_{i}^{\prime}+1 / 2(i=1,2,3)$. At last, the diabatic coupling $H_{c}$ is taken to be the only first-order term authorized by symmetry, that is

$$
H_{c}=\lambda q_{3} \text {. }
$$

Calculation of the $\left\langle v_{1}^{\prime}, v_{2}^{\prime}, v_{3}^{\prime}\left|H_{c}\right| v_{1}, v_{2}, v_{3}\right\rangle$ integrals is the numerically difficult step in the process of building the full Hamiltonian matrix in the $\left|v_{1}, v_{2}, v_{3}\right\rangle$ and $\left|v_{1}^{\prime}, v_{2}^{\prime}, v_{3}^{\prime}\right\rangle$ bases. In addition to the parameter $\lambda$, these integrals depend only on the relationship between the $\left(q_{1}, q_{2}, q_{3}\right)$ and $\left(q_{1}^{\prime}, q_{2}^{\prime}, q_{3}^{\prime}\right)$ sets of normal coordinates, which, as in Ref. 14, is taken in the form,

$$
\begin{aligned}
\left(\begin{array}{l}
q_{1}^{\prime} \\
q_{2}^{\prime} \\
q_{3}^{\prime}
\end{array}\right) & =\mathbf{A}\left(\begin{array}{l}
q_{1} \\
q_{2} \\
q_{3}
\end{array}\right)+\mathbf{B} \\
& =\left(\begin{array}{ccc}
0.899 & -0.532 & 0 \\
0.301 & 0.906 & 0 \\
0 & 0 & 0.693
\end{array}\right)\left(\begin{array}{l}
q_{1} \\
q_{2} \\
q_{3}
\end{array}\right)+\left(\begin{array}{c}
1.100 \\
-5.730 \\
0
\end{array}\right) .
\end{aligned}
$$

This relationship was kept fixed in all of our calculations, although the fundamental frequencies were allowed to vary slightly around the values, which were used to obtain Eq. (2.6). This is of little practical consequence, because the equilibrium geometry of the excited surface is anyway still rather poorly known. ${ }^{4}$ Since the numerical calculation of these integrals is one of the fundamental issues for this study, the procedure we used to calculate them is sketched in the Appendix.

As already noted in the Introduction, the principal problem, which arises when using Eqs. (2.1)-(2.6) to build and diagonalize the Hamiltonian matrix, is the size of the total harmonic basis. Indeed, this basis must contain several tens of thousands of vectors if the spectrum is to be converged up to 11000 or $12000 \mathrm{~cm}^{-1}$ above the quantum mechanical ground state. This size being not compatible with the large number of iterations required by an adjustment procedure, we had to work with a more suitable basis, obtained from first order perturbation theory. The procedure for determining this basis, as well as the calculation of the matrix elements of $\mathbf{H}$ in the new basis, are described in some detail in the following section.

\section{THE PERTURBATIVE BASIS}

Let us note $E_{n}^{g}$ and $E_{n}^{e}$ the eigenvalues of the uncoupled Hamiltonians $H_{g}$ and $H_{e}$ and $\varphi_{n}^{g}$ and $\varphi_{n}^{e}$ the associated eigenvectors, 


$$
\begin{aligned}
& H_{g} \varphi_{n}^{g}=E_{n}^{g} \varphi_{n}^{g}, \\
& H_{e} \varphi_{n}^{e}=E_{n}^{e} \varphi_{n}^{e} .
\end{aligned}
$$

The eigenvectors of the full Hamiltonian $\mathbf{H}$ of Eq. (2.1) are obtained, at first order of perturbation theory, in the form

$$
\left(\begin{array}{c}
\sum_{k} \frac{\left\langle\varphi_{k}^{e}\left|H_{c}\right| \varphi_{n}^{g}\right\rangle}{E_{n}^{g}-E_{k}^{e}} \varphi_{k}^{e} \\
\varphi_{n}^{g}
\end{array}\right),
$$

for the eigenvectors of $\mathbf{H}$ built on the eigenvectors of $H_{g}$, and

$$
\left(\begin{array}{c}
\varphi_{n}^{e} \\
\sum_{k} \frac{\left\langle\varphi_{n}^{e}\left|H_{c}\right| \varphi_{k}^{g}\right\rangle}{E_{n}^{e}-E_{k}^{g}} \varphi_{k}^{g}
\end{array}\right)
$$

for those built on the eigenvectors of $H_{e}$. It is well-known, that the vectors in Eqs. (3.2)-(3.3) are good approximations of the eigenvectors of $\mathbf{H}$ if (and only if) the ratios which appear in these equations remain small, that is, if the zeroorder energies $E_{n}^{g}$ of the ground electronic surface are not accidentally close to the zero-order energies $E_{n}^{e}$ of the excited electronic surface. If this condition is not fulfilled, that is, if there exist accidental resonances between specific pairs of states belonging to different electronic surfaces, then the approximation breaks down. It is therefore interesting to build new bases of vectors $\psi_{n}^{g}$ and $\psi_{n}^{e}$, such that

$$
\psi_{n}^{g}=\left(\begin{array}{c}
\sum_{k} \alpha_{n k} \varphi_{k}^{e} \\
\varphi_{n}^{g}
\end{array}\right), \quad \psi_{n}^{e}=\left(\begin{array}{c}
\varphi_{n}^{e} \\
\sum_{k} \beta_{n k} \varphi_{k}^{g}
\end{array}\right),
$$

where the coefficients $\alpha_{n k}$ and $\beta_{n k}$ are defined as follows:

$$
\begin{aligned}
& \text { if }\left|\frac{\left\langle\varphi_{k}^{e}\left|H_{c}\right| \varphi_{n}^{g}\right\rangle}{E_{n}^{g}-E_{k}^{e}}\right| \leqslant \varepsilon, \\
& \text { then } \alpha_{n k}=\frac{\left\langle\varphi_{k}^{e}\left|H_{c}\right| \varphi_{n}^{g}\right\rangle}{E_{n}^{g}-E_{k}^{e}} \text { else } \alpha_{n k}=0, \\
& \text { if }\left|\frac{\left\langle\varphi_{n}^{e}\left|H_{c}\right| \varphi_{k}^{g}\right\rangle}{E_{n}^{e}-E_{k}^{g}}\right| \leqslant \varepsilon, \\
& \text { then } \beta_{n k}=\frac{\left\langle\varphi_{n}^{e}\left|H_{c}\right| \varphi_{k}^{g}\right\rangle}{E_{n}^{e}-E_{k}^{g}} \text { else } \beta_{n k}=0 .
\end{aligned}
$$

In Eq. (3.5), $\varepsilon$ is a threshold parameter, which separates resonantly (large ratios) from nonresonantly (small ratios) coupled pairs of zero-order eigenstates. We used $\varepsilon=0.05$ in the calculations reported in this article but, of course, the final result does not depend on the precise value of $\varepsilon$. The matrix elements of $\mathbf{H}$ in the new basis are next obtained in the form,

$$
\begin{aligned}
\left\langle\psi_{m}^{g}|\mathbf{H}| \psi_{n}^{g}\right\rangle= & \delta_{m n} E_{n}^{g}+\sum_{k}\left(\alpha_{m k} \alpha_{n k} E_{k}^{e}+\alpha_{m k}\left\langle\varphi_{k}^{e}\left|H_{c}\right| \varphi_{n}^{g}\right\rangle\right. \\
& \left.+\alpha_{n k}\left\langle\varphi_{k}^{e}\left|H_{c}\right| \varphi_{m}^{g}\right\rangle\right), \\
\left\langle\psi_{m}^{e}|\mathbf{H}| \psi_{n}^{e}\right\rangle= & \delta_{m n} E_{n}^{e}+\sum_{k}\left(\beta_{m k} \beta_{n k} E_{k}^{g}+\beta_{m k}\left\langle\varphi_{n}^{e}\left|H_{c}\right| \varphi_{k}^{g}\right\rangle\right. \\
& \left.+\beta_{n k}\left\langle\varphi_{m}^{e}\left|H_{c}\right| \varphi_{k}^{g}\right\rangle\right), \\
\left\langle\psi_{m}^{e}|\mathbf{H}| \psi_{n}^{g}\right\rangle= & \left\langle\varphi_{m}^{e}\left|H_{c}\right| \varphi_{n}^{g}\right\rangle+\alpha_{n m} E_{m}^{e}+\beta_{m n} E_{n}^{g} \\
& +\sum_{k} \sum_{j} \alpha_{n j} \beta_{m k}\left\langle\varphi_{j}^{e}\left|H_{c}\right| \varphi_{k}^{g}\right\rangle,
\end{aligned}
$$

where $\delta_{m n}$ is Kronecker's symbol. The last equation in Eq. (3.6) shows that, in the new basis of vectors $\psi_{n}^{g}$ and $\psi_{n}^{e}$, the nonadiabatic coupling $\left\langle\psi_{m}^{e}|\mathbf{H}| \psi_{n}^{g}\right\rangle$ between two vectors belonging to different surfaces is small, except when the zeroorder energies of these vectors are accidentally resonant. In this later case, the off-diagonal matrix element is close to the unperturbed one, that is to $\left\langle\varphi_{m}^{e}\left|H_{c}\right| \varphi_{n}^{g}\right\rangle$. Consequently, the size of the matrix one has to build to obtain a spectrum converged up to a given energy is of much smaller dimensions than the size required with the harmonic basis. For example, this size is typically of the order of 500-1000 to converge the spectrum up to $11800 \mathrm{~cm}^{-1}$.

Before concluding this section, it is perhaps not completely useless to emphasize that the basis defined by Eqs. (3.4)-(3.5) is neither orthogonal nor normalized, so that the energy levels and the quantum states of $\mathbf{H}$ are not obtained as the eigenvalues and eigenvectors of the Hamiltonian matrix computed in this basis. In the perturbative basis, one has instead to solve the slightly more complex generalized eigenproblem,

$$
\mathbf{H} \psi=E \mathbf{N} \psi,
$$

where $\mathbf{N}$ is the matrix of the overlaps $\left\langle\psi_{m}^{g} \mid \psi_{n}^{g}\right\rangle,\left\langle\psi_{m}^{e} \mid \psi_{n}^{e}\right\rangle$, and $\left\langle\psi_{m}^{e} \mid \psi_{n}^{g}\right\rangle$ (N is equal to the identity matrix in the case of an orthonormalized basis). A numerically efficient method for solving Eq. (3.7) consists in finding the matrices $\mathbf{V}$ and $\boldsymbol{\Lambda}$ of, respectively, the eigenvectors and eigenvalues of $\mathbf{N}$,

$$
\mathbf{N V}=\mathbf{V} \Lambda
$$

and in calculating the matrix $\mathbf{M}$,

$$
\mathbf{M}=\mathbf{V} \boldsymbol{\Lambda}^{-1 / 2 t} \mathbf{V}
$$

( $\mathbf{M}$ is also equal to the identity matrix in the case of an orthonormalized basis). Simple calculations then show that the quantized energies of Eq. (3.7) are the eigenvalues of M H M, while the matrix of the generalized eigenvectors of Eq. (3.7) is equal to the matrix of the eigenvectors of $\mathbf{M ~ H ~ M ~}$ multiplied, on the left-hand side, by $\mathbf{M}$.

\section{CALCULATIONS, RESULTS, AND DISCUSSION}

To summarize, actual calculations are performed as follows. The initial basis is first determined by retaining all the vectors $\left|v_{1}, v_{2}, v_{3}\right\rangle$ and $\left|v_{1}^{\prime}, v_{2}^{\prime}, v_{3}^{\prime}\right\rangle$, such that 


$$
\begin{aligned}
& 1337 v_{1}+758 v_{2}+1672 v_{3} \leqslant E_{\max }^{g}, \\
& 9700+1310 v_{1}^{\prime}+745 v_{2}^{\prime}+800 v_{3}^{\prime} \leqslant E_{\text {max }}^{e} .
\end{aligned}
$$

For the purpose of calculating the spectrum up to 11800 $\mathrm{cm}^{-1}$ above the quantum mechanical ground state, suitable values for the upper limits are $E_{\max }^{g}=30000 \mathrm{~cm}^{-1}$ and $E_{\max }^{e}=50000 \mathrm{~cm}^{-1}$, which leads to basis sizes of 3182 and 15504 for, respectively, the ground and excited surfaces.

The eigenstates of the uncoupled Hamiltonians $H_{g}$ and $H_{e}$ are then evaluated for each new set of parameters of the effective Hamiltonian. The eigenvectors $\varphi_{n}^{e}$ of $H_{e}$ are obviously just the $\left|v_{1}^{\prime}, v_{2}^{\prime}, v_{3}^{\prime}\right\rangle$ vectors, while the corresponding eigenvalues $E_{n}^{e}$ are calculated according to Eq. (2.4). In contrast, calculation of the functions $\varphi_{n}^{g}$ and the energies $E_{n}^{g}$ for the ground electronic surface requires some more effort, since these quantities are obtained from the diagonalization of small matrices of $\left|v_{1}, v_{2}, v_{3}\right\rangle$ vectors coupled by the $3 \omega_{1} \approx \omega_{2}+2 \omega_{3}$ resonance of Eq. (2.3).

The perturbative basis, which consists of the vectors $\psi_{n}^{g}$ and $\psi_{n}^{e}$ of Eqs. (3.4)-(3.5), is next evaluated. One retains in this basis all the vectors $\psi_{n}^{g}$ and $\psi_{n}^{e}$, which are built on vectors $\varphi_{n}^{g}$ and $\varphi_{n}^{e}$ with associated zero-order energies $E_{n}^{g}$ and $E_{n}^{e}$ smaller than a given threshold $E_{\max }$ (relative to the minimum of the ground electronic surface). In order to check the convergence of the calculations, the results obtained with two different values of $E_{\max }$ are compared, namely, $E_{\max }$ $=16000 \mathrm{~cm}^{-1}$ (about 500 vectors for the ground surface and 40 vectors for the excited surface) and $E_{\max }=20000 \mathrm{~cm}^{-1}$ (about 1200 vectors for the ground surface and 200 vectors for the excited surface). Note that, according to Eqs. (3.4)(3.5), each vector $\psi_{n}^{g}$ depends, in addition to $\varphi_{n}^{g}$, on the 15504 vectors $\left|v_{1}^{\prime}, v_{2}^{\prime}, v_{3}^{\prime}\right\rangle$, while each vector $\psi_{n}^{e}$ depends, in addition to $\varphi_{n}^{e}$, on the 3182 vectors $\left|v_{1}, v_{2}, v_{3}\right\rangle$.

At last, the Hamiltonian matrix is built according to Eq. (3.6) and the quantized states of $\mathbf{H}$ are obtained from the procedure sketched at the end of Sec. III. Eventually, the eigenvectors of $\mathbf{H}$ are expressed back in terms of the vectors $\left|v_{1}, v_{2}, v_{3}\right\rangle$ and $\left|v_{1}^{\prime}, v_{2}^{\prime}, v_{3}^{\prime}\right\rangle$. After comparison of the calculated and experimental spectra, the standard gradient method is used to minimize the computed root-mean-square (rms) error.

The experimental spectrum up to $11800 \mathrm{~cm}^{-1}$ above the quantum mechanical ground state (against $11400 \mathrm{~cm}^{-1}$ in Ref. 14) was used to adjust the parameters of the effective Hamiltonian. This energy window contains 298 states with predominant $X^{2} A_{1}$ ground electronic character and nine states with predominant $A^{2} B_{2}$ excited electronic character, of which only 1 state with predominant $A^{2} B_{2}$ excited electronic character $([1,0,1])$ has not been observed experimentally. In addition to energy, the experimental spectrum also provides a clear-cut determination of the total vibronic symmetry $\left(A_{1}\right.$ or $\left.B_{2}\right)$ of the recorded states. ${ }^{1-4}$ The vibrational terms (i.e., the rotationless band origins) of states with $A_{1}$ vibronic symmetry are directly obtained from the recorded laser induced dispersed fluorescence (LIDFS) spectra. In contrast, the lowest rotational level of states with $B_{2}$ vibronic symmetry observed in LIDFS spectra is $N=K=1$. For the purpose of comparison with calculated energies, these ob-
TABLE I. Adjusted values (second column) and standard deviations (third column) for the parameters of the effective Hamiltonian of Eqs. (2.1)-(2.6).

\begin{tabular}{|c|c|c|}
\hline & Value & Uncertainty \\
\hline$\omega_{1}$ & 1357.4090 & 3.2911 \\
\hline$\omega_{2}$ & 756.8245 & 0.8396 \\
\hline$\omega_{3}$ & 1670.3878 & 0.9327 \\
\hline$x_{11}$ & -11.9870 & 1.5479 \\
\hline$x_{12}$ & -5.0272 & 0.4345 \\
\hline$x_{13}$ & -28.9949 & 0.2445 \\
\hline$x_{22}$ & 0.0763 & 0.1547 \\
\hline$x_{23}$ & -10.4319 & 0.2975 \\
\hline$x_{33}$ & -14.7341 & 0.1949 \\
\hline$y_{111}$ & 1.4238 & 0.2577 \\
\hline$y_{112}$ & 0.3033 & 0.1512 \\
\hline$y_{122}$ & -0.2087 & 0.0226 \\
\hline$y_{222}$ & -0.0363 & 0.0075 \\
\hline$y_{233}$ & -0.1933 & 0.0477 \\
\hline$z_{1111}$ & -0.0892 & 0.0137 \\
\hline$z_{1112}$ & -0.0558 & 0.0148 \\
\hline$z_{1113}$ & -0.2460 & 0.0071 \\
\hline$z_{1123}$ & -0.1306 & 0.0230 \\
\hline$z_{1223}$ & -0.0590 & 0.0162 \\
\hline$z_{2233}$ & 0.0186 & 0.0026 \\
\hline$k$ & 0.8360 & 0.1815 \\
\hline$k_{1}$ & -0.1710 & 0.0561 \\
\hline$E_{0}^{\prime}$ & 10209.0874 & 20.2592 \\
\hline$\omega_{1}^{\prime}$ & 1315.0927 & 9.5659 \\
\hline$\omega_{2}^{\prime}$ & 766.2419 & 12.0323 \\
\hline$\omega_{3}^{\prime}$ & 753.1696 & 17.9987 \\
\hline$x_{12}^{\prime}$ & -36.9088 & 6.3631 \\
\hline$x_{23}^{\prime}$ & -43.3650 & 8.2839 \\
\hline$x_{33}^{\prime}$ & 27.9314 & 4.6044 \\
\hline$\lambda$ & 331.7647 & 18.8067 \\
\hline
\end{tabular}
All values are expressed in $\mathrm{cm}^{-1}$.

served energies are extrapolated down to $N=K=0$ by subtracting from the observed energies the rotational constant $A+\bar{B}$, where $A$ and $\bar{B}$ are estimated according to Eqs. (1) and (2) of Ref. 3.

A set of 30 converged parameters (22 parameters for $H_{g}, 7$ parameters for $H_{e}$, and 1 parameter for $H_{c}$ ) is shown in Table I. The rms and maximum errors between the measured energies and the calculated ones are $3.8 \mathrm{~cm}^{-1}$ and 19.7 $\mathrm{cm}^{-1}$, respectively, for the 306 experimentally observed states. Fit residuals are plotted in Fig. 1(a) as a function of energy. Note that the small uncertainties reported in Table I for the parameters of $H_{e}$, as well as the small errors for the calculated energies of the eight states with predominant $A^{2} B_{2}$ excited electronic character, are largely meaningless since, for this surface, the number of parameters (7) is very close to the number of experimental data taken into account (8).

One of the most interesting information of Table I is the value for the coupling parameter $\lambda \approx 332 \mp 19 \mathrm{~cm}^{-1}$. The main reason, why this estimation is substantially smaller than the estimation of Ref. $14\left(\lambda \approx 600 \mathrm{~cm}^{-1}\right)$ is, as already stated in the Introduction, that the overlap matrix elements used in this first work were not precise enough, because of the loss of an unexpectedly large number of accuracy digits (several tens of them) following near cancellation of very large numbers. The overlap integrals were calculated to, roughly, only one half of their actual values, which resulted in an estima- 


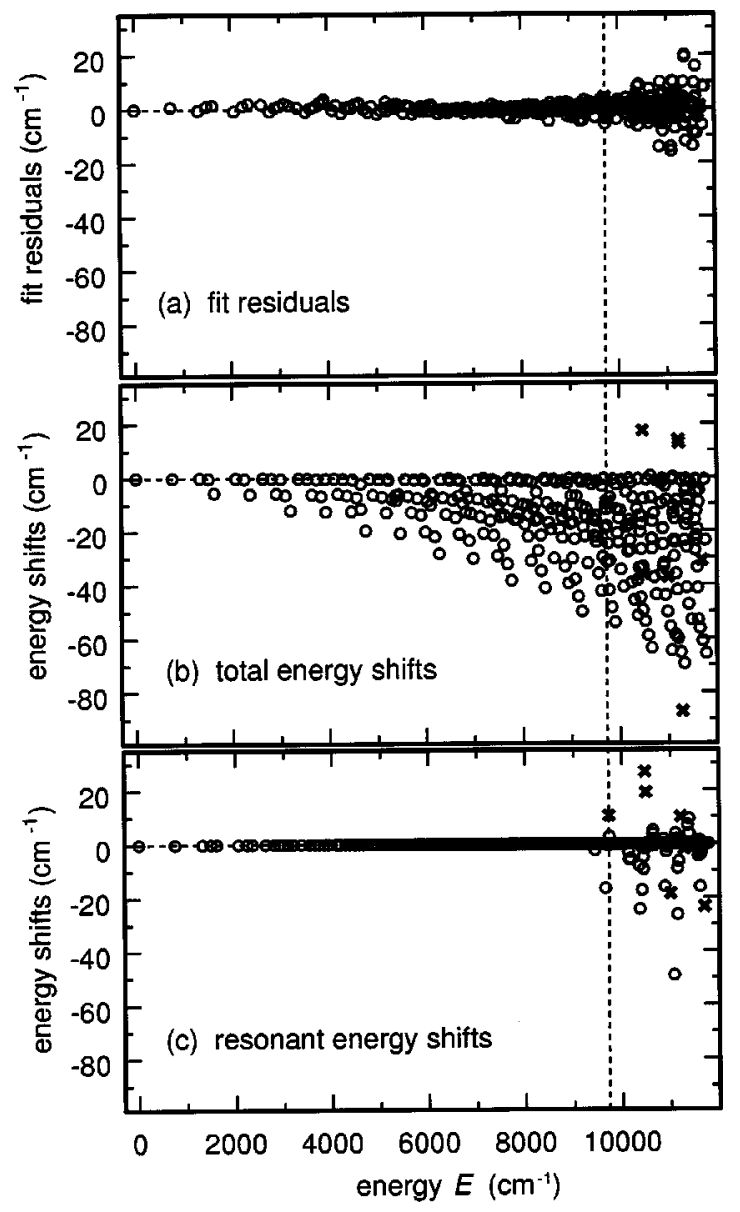

FIG. 1. (a) Plot of the fit residuals as a function of the experimentally determined energies up to $11800 \mathrm{~cm}^{-1}$ above the quantum mechanical ground state. The fit residuals are defined as the difference between the experimentally determined energies and the eigenvalues of the effective Hamiltonian of Eqs. (2.1)-(2.6) and Table I. (b) Plot of the calculated total energy shifts caused by the diabatic coupling $H_{c}$ as a function of the experimentally determined energies. The total energy shifts are defined as the difference between the eigenvalues of the effective Hamiltonian of Eqs. (2.1)-(2.6) and Table I and the eigenvalues obtained when setting $\lambda=0$. (c) Plot of the calculated resonant energy shifts caused by the diabatic coupling $H_{c}$ as a function of the experimentally determined energies. The resonant energy shifts are defined as the difference between the eigenvalues of the effective Hamiltonian of Eqs. (2.1)-(2.6) and Table I and the eigenvalues obtained when setting all $\left\langle\psi_{m}^{e}|\mathbf{H}| \psi_{n}^{g}\right\rangle$ elements to zero in the Hamiltonian matrix expressed in the perturbative basis [a value $\varepsilon=0.05$ is assumed in the definitions of Eq. (3.5)]. In all plots, open circles denote states with predominant $X^{2} A_{1}$ ground electronic character, while crosses $(\times)$ denote states with predominant $A^{2} B_{2}$ excited electronic character. The vertical lines indicate the energy of the vibrational ground state of the excited electronic surface. Note also that both the coordinate and abscissa scales are identical for the three plots.

tion for $\lambda$ too large by a factor of about 2 . It is stressed, once more, that the determination of $\lambda$ does not rely on the total energy shifts caused by $H_{c}=\lambda q_{3}$, but only on a small part of these quantities, i.e., the resonant shifts. This point is clearly visualized in Figs. 1(b) and 1(c), which show, respectively, the total and resonant energy shifts caused by $H_{c}$. The total energy shifts of Fig. 1(b) are obtained by subtracting the energies calculated with $\lambda=0$ from the energies calculated with the parameters of Table I. Although this figure is somewhat obscured by the fact that the shifts for all of the states are displayed on the same plot, it is nevertheless clear that the largest part of these shifts increases regularly with quantum numbers and energy. We actually showed in Refs. 15 and 16 that the regular part of the energy shifts is due to the nonresonant couplings, that is, to the interactions between states of different surfaces, which are well separated in energy. We also showed that these nonresonant shifts cannot be distinguished from the anharmonicities inside each surface (hence the above remark that they are of no use for the determination of the coupling parameter $\lambda$ ). The principal reason, why the number of vectors required to converge the spectrum is much smaller with the perturbative basis of Sec. III than with the harmonic basis, is precisely that the perturbative basis for each surface takes into account, by construction, the nonresonant shifts experienced by the states of this surface. Therefore, the resonant shifts (i.e., the shifts resulting from the interaction between states of different surfaces which are almost degenerate in energy) can be obtained as the difference between the eigenvalues of the effective Hamiltonian of Eqs. (2.1)-(2.6) and the eigenvalues obtained when setting all $\left\langle\psi_{m}^{e}|\mathbf{H}| \psi_{n}^{g}\right\rangle$ elements to zero in the Hamiltonian matrix expressed in the perturbative basis. The result obtained with $\varepsilon=0.05$ is plotted in Fig. 1(c). It is seen that, in contrast with nonresonant ones, resonant shifts are significant only for a limited number of states. The present determination of $\lambda$ actually relies on these (relatively few) resonant shifts and the resulting increase of precision in the calculated energies. It is therefore not excluded that this estimation for $\lambda$ might still vary to some extent if the working data set is extended to higher energies. It turns out that the present estimation $\lambda \approx 332 \mp 19 \mathrm{~cm}^{-1}$ is of the same order of magnitude as the first experimental determination of Delon and Jost $(\lambda \approx 280 \mp 50) \mathrm{cm}^{-1},{ }^{1}$ which was obtained from approximate calculations performed on a much more limited data set. In contrast, this value is much smaller than the estimations derived from recent ab initio calculations, which range from $700 \mathrm{~cm}^{-1}$ (Ref. 8) to more than $2400 \mathrm{~cm}^{-1} \cdot{ }^{10}$ As stated in Ref. 8 , the reason for such an uncertainty in the determination of $\lambda$ is "not clear at that point."

This work and the previous one in Ref. 14 enable a better understanding of the experimental spectrum of $\mathrm{NO}_{2}$ up to $11800 \mathrm{~cm}^{-1}$ above the quantum mechanical ground state. Indeed, several states which were left unassigned in Table VI of Ref. 3 could be safely assigned in the course of this work, while some additional states could be retrieved in the recorded spectra thank to their calculated energies (this procedure eventually led to the reassignment of a few states). This is the first reason, why the energies of the states observed above $9500 \mathrm{~cm}^{-1}$ are reported in Table II together with their calculated decomposition on the harmonic oscillator bases. The description of states with lower energy can be found in Refs. 1 and 3. The second reason for reporting the computed eigenvectors in Table II is that, within the approximation of the effective Hamiltonian of Eqs. (2.1)-(2.6), the only important coupling observed below $9500 \mathrm{~cm}^{-1}$ is due to the approximate $3 \omega_{1} \approx \omega_{2}+2 \omega_{3}$ vibrational resonance of Eq. (2.3), while additional couplings due to $H_{c}$ appear above $9500 \mathrm{~cm}^{-1}$. Among these new couplings, the most important one is of course the resonant vibronic coupling between states of different surfaces, which gives rise to the resonant 
TABLE II. $\mathrm{NO}_{2}$ vibronic states located between 9500 and $11800 \mathrm{~cm}^{-1}$ above the quantum mechanical ground state. The first column indicates the rank of the state in a given electronic surface (the quantum mechanical ground state of each surface is \#1). The second column shows, for states with $B_{2}$ vibronic symmetry, the energy (in $\mathrm{cm}^{-1}$ above the quantum mechanical ground state) of the $N=K=1$ rotational component obtained from LIDFS experiments. The rotationless band origins are shown in the third column. For most states with $B_{2}$ vibronic symmetry, the band origin is obtained by subtracting the rotational constant $A+\bar{B}$ [estimated according to Eqs. (1) and (2) Ref. 3] from the $N=K=1$ energy reported in the second column. For states with $A_{1}$ vibronic symmetry, the energy of the band origin is directly obtained from LIDFS experiments. The fourth column indicates the difference between the "experimental" energy of column 3 and the energy obtained from the effective Hamiltonian of Eqs. (2.1)-(2.6) and the parameters of Table I. The fifth column shows the calculated probability (in percents) for finding the state in the diabatic excited electronic surface $A^{2} B_{2}$. A more precise description of the wave function is provided in the last column, which indicates its decomposition on the harmonic bases of the $X^{2} A_{1}$ (normal brackets) and $A^{2} B_{2}$ (square brackets) diabatic electronic surfaces. All calculated contributions larger than 0.1 are shown.

\begin{tabular}{|c|c|c|c|c|c|}
\hline No. & $\begin{array}{c}\begin{array}{c}\text { Energy } \\
\left(\mathrm{cm}^{-1}\right)\end{array} \\
K=N=1\end{array}$ & $\begin{array}{c}\begin{array}{c}\text { Energy } \\
\left(\mathrm{cm}^{-1}\right)\end{array} \\
K=N=0\end{array}$ & $\begin{array}{c}\text { obs. }- \text { calc. } \\
\text { error } \\
\left(\mathrm{cm}^{-1}\right)\end{array}$ & $\% A^{2} B_{2}$ & Eigenvector decomposition \\
\hline 165 & 9510.20 & 9499.98 & -0.08 & 0.16 & $0.715(1,5,3)-0.697(4,4,1)$ \\
\hline 166 & & 9512.15 & 0.26 & 0.02 & $0.979(4,2,2)-0.199(1,3,4)$ \\
\hline 167 & 9529.28 & 9518.12 & 1.00 & 0.18 & $0.716(4,4,1)+0.695(1,5,3)$ \\
\hline 168 & & 9524.46 & 1.33 & 0.03 & $0.994(4,6,0)+0.103(1,7,2)$ \\
\hline 169 & 9539.85 & 9531.82 & -3.34 & 0.00 & $0.996(4,0,3)$ \\
\hline 170 & & 9561.57 & 1.51 & 0.20 & $0.978(1,3,4)+0.199(4,2,2)$ \\
\hline 171 & 9631.80 & 9624.27 & 2.85 & 0.04 & $0.995(1,1,5)$ \\
\hline 172 & & 9640.77 & 2.13 & 0.22 & $0.998(0,13,0)$ \\
\hline 173 & 9667.96 & 9653.79 & 1.10 & 17.31 & $0.902(0,11,1)+0.408[0,0,0]$ \\
\hline 174 & & 9672.77 & 0.98 & 0.00 & $0.999(7,1,0)$ \\
\hline 175 & & 9697.32 & 0.13 & 0.75 & $0.968(0,9,2)-0.225(3,8,0)$ \\
\hline 176 & 9725.87 & 9714.10 & 1.45 & 2.82 & $0.941(3,6,1)-0.273(0,7,3)+0.163[0,0,0]$ \\
\hline 177 & & 9717.28 & -1.19 & 0.13 & $0.974(3,8,0)+0.223(0,9,2)$ \\
\hline 178 & & 9732.21 & 3.69 & 0.10 & $0.983(3,4,2)-0.173(0,5,4)$ \\
\hline 1 & 9738.20 & 9733.50 & 4.28 & 59.92 & $0.773[0,0,0]+0.482(0,7,3)-0.383(0,11,1)$ \\
\hline 179 & 9747.29 & 9735.76 & -5.73 & 18.18 & $0.826(0,7,3)-0.420[0,0,0]+0.331(3,6,1)+0.145(0,11,1)$ \\
\hline 180 & 9762.81 & 9753.87 & -3.63 & 0.04 & $0.992(3,2,3)-0.118(0,3,5)$ \\
\hline 181 & & 9781.28 & -1.00 & 0.45 & $0.981(0,5,4)+0.173(3,4,2)$ \\
\hline 182 & 9806.85 & 9796.43 & 0.19 & 0.00 & $0.999(6,1,1)$ \\
\hline 183 & & 9796.99 & -3.81 & 0.00 & $0.997(3,0,4)$ \\
\hline 184 & 9845.21 & 9836.87 & 0.36 & 0.33 & $0.989(0,3,5)+0.118(3,2,3)$ \\
\hline 185 & & 9856.44 & 2.00 & 0.00 & $1.000(6,3,0)$ \\
\hline 186 & & 9905.43 & -1.05 & 0.09 & $0.995(0,1,6)$ \\
\hline 187 & & 9920.66 & 0.38 & 0.25 & $0.998(2,10,0)$ \\
\hline 188 & 9941.20 & 9928.51 & -0.47 & 1.21 & $0.993(2,8,1)$ \\
\hline 189 & & 9950.91 & 2.18 & 0.32 & $0.996(2,6,2)$ \\
\hline 190 & 9985.29 & 9975.53 & -4.05 & 0.18 & $0.966(2,4,3)-0.249(5,3,1)$ \\
\hline 191 & & 9984.01 & 0.14 & 0.00 & $0.989(5,1,2)-0.139(2,2,4)$ \\
\hline 192 & 10017.80 & 10006.31 & 3.20 & 0.03 & $0.968(5,3,1)+0.248(2,4,3)$ \\
\hline 193 & & 10025.87 & 0.47 & 0.08 & $0.988(2,2,4)+0.139(5,1,2)$ \\
\hline 194 & & 10043.60 & 2.68 & 0.02 & $0.999(5,5,0)$ \\
\hline 195 & 10089.66 & 10082.40 & 0.32 & 0.00 & $0.998(2,0,5)$ \\
\hline 196 & & 10132.64 & -0.25 & 1.19 & $0.988(1,12,0)$ \\
\hline 197 & 10159.36 & 10145.36 & -1.82 & 3.58 & $0.979(1,10,1)-0.161[0,1,0]$ \\
\hline 198 & & 10174.63 & 3.75 & 1.49 & $0.979(1,8,2)-0.110(1,12,0)$ \\
\hline 199 & & 10185.29 & 1.81 & 0.00 & $0.999(8,0,0)$ \\
\hline 200 & 10209.84 & 10198.93 & 0.70 & 0.30 & $0.736(1,6,3)-0.672(4,5,1)$ \\
\hline 201 & & 10203.93 & 2.02 & 0.06 & $0.972(4,3,2)-0.226(1,4,4)$ \\
\hline 202 & 10222.40 & 10213.62 & -2.60 & 0.01 & $0.981(4,1,3)-0.114(1,2,5)+0.112(7,0,1)$ \\
\hline 203 & 10228.12 & 10216.13 & -0.43 & 0.39 & $0.736(4,5,1)+0.664(1,6,3)-0.101(4,1,3)$ \\
\hline 204 & & 10232.65 & 0.50 & 0.13 & $0.995(4,7,0)$ \\
\hline 205 & & 10252.54 & 1.79 & 0.33 & $0.970(1,4,4)+0.226(4,3,2)$ \\
\hline 206 & 10280.32 & 10270.61 & -5.37 & 0.00 & $0.993(7,0,1)-0.109(4,1,3)$ \\
\hline 207 & 10312.69 & 10304.70 & 2.39 & 0.13 & $0.990(1,2,5)+0.119(4,1,3)$ \\
\hline 208 & & 10352.46 & -4.33 & 8.58 & $0.919(0,14,0)+0.285[0,0,1]+0.235(0,10,2)$ \\
\hline 209 & 10377.37 & 10361.78 & -3.95 & 20.26 & $0.870(0,12,1)-0.440[0,1,0]-0.105(1,10,1)+0.102(0,8,3)$ \\
\hline 210 & & 10371.74 & 3.56 & 0.00 & $0.999(7,2,0)$ \\
\hline 211 & & 10375.49 & 5.50 & 0.01 & $0.998(1,0,6)$ \\
\hline 212 & & 10403.19 & 8.16 & 12.35 & $0.854(0,10,2)-0.347(0,14,0)+0.335[0,0,1]$ \\
\hline 213 & 10424.80 & 10412.23 & -1.97 & 1.65 & $0.953(3,7,1)-0.242(0,8,3)-0.114(0,12,1)-0.112[0,1,0]$ \\
\hline 214 & & 10415.65 & -6.51 & 0.64 & $0.947(3,5,2)+0.246(3,9,0)-0.166(0,6,4)$ \\
\hline 215 & & 10435.48 & 8.77 & 2.55 & $0.945(3,9,0)-0.254(3,5,2)+0.157[0,0,1]$ \\
\hline 216 & 10449.22 & 10436.67 & -1.35 & 7.30 & $0.901(0,8,3)-0.268(0,12,1)-0.253[0,1,0]+0.164(3,7,1)$ \\
\hline 217 & 10451.92 & 10442.40 & -0.95 & 0.10 & $0.989(3,3,3)-0.134(0,4,5)$ \\
\hline 218 & & 10446.07 & 3.59 & 0.00 & $0.999(6,0,2)$ \\
\hline 219 & & 10477.36 & -0.82 & 0.02 & $0.994(3,1,4)$ \\
\hline
\end{tabular}


TABLE II. (Continued.)

\begin{tabular}{|c|c|c|c|c|c|}
\hline No. & $\begin{array}{c}\begin{array}{c}\text { Energy } \\
\left(\mathrm{cm}^{-1}\right)\end{array} \\
K=N=1\end{array}$ & $\begin{array}{c}\text { Energy } \\
\left(\mathrm{cm}^{-1}\right) \\
K=N=0\end{array}$ & $\begin{array}{l}\text { obs. }- \text { calc. } \\
\text { error } \\
\left(\mathrm{cm}^{-1}\right)\end{array}$ & $\% A^{2} B_{2}$ & Eigenvector decomposition \\
\hline 220 & & 10478.83 & 1.89 & 9.79 & $\begin{array}{l}0.894(0,6,4)+0.303[0,0,1]-0.228(0,10,2)+0.141(3,5,2)- \\
0.106(3,9,0)\end{array}$ \\
\hline 2 & 10483.60 & 10478.00 & -4.02 & 62.98 & $\begin{array}{l}0.791[0,1,0]+0.378(0,12,1)+0.318(0,8,3)+0.232(3,7,1)- \\
0.177(2,9,1)+0.120(1,10,1)-0.102(1,11,1)\end{array}$ \\
\hline 221 & 10498.19 & 10486.38 & 1.09 & 0.04 & $0.999(6,2,1)$ \\
\hline 3 & & 10490.20 & 0.46 & 59.47 & $\begin{array}{l}0.768[0,0,1]-0.389(0,6,4)-0.361(0,10,2)-0.182(3,9,0)- \\
0.151(0,14,0)+0.125(2,11,0)-0.114(3,5,2)\end{array}$ \\
\hline 222 & 10532.80 & 10523.43 & -0.29 & 0.55 & $0.985(0,4,5)+0.134(3,3,3)$ \\
\hline 223 & & 10557.41 & 2.96 & 0.01 & $1.000(6,4,0)$ \\
\hline 224 & & 10583.21 & 1.31 & 0.25 & $0.992(0,2,6)$ \\
\hline 225 & & 10633.06 & -5.47 & 2.03 & $0.986(2,11,0)-0.134[0,0,1]$ \\
\hline 226 & 10649.26 & 10635.65 & -5.56 & 4.34 & $0.975(2,9,1)+0.185[0,1,0]$ \\
\hline 227 & & 10654.47 & 7.19 & 1.98 & $0.986(2,7,2)-0.105[0,0,1]$ \\
\hline 228 & 10663.43 & 10656.54 & -3.79 & 0.02 & $0.997(0,0,7)$ \\
\hline 229 & 10669.13 & 10660.61 & -2.00 & 0.00 & $0.997(5,0,3)$ \\
\hline 230 & 10677.46 & 10667.12 & -2.62 & 0.37 & $0.965(2,5,3)-0.248(5,4,1)$ \\
\hline 231 & & 10670.56 & 4.17 & 0.02 & $0.983(5,2,2)-0.169(2,3,4)$ \\
\hline 232 & 10708.54 & 10695.97 & 0.22 & 0.08 & $0.968(5,4,1)+0.246(2,5,3)$ \\
\hline 233 & & 10707.27 & -0.79 & 0.17 & $0.983(2,3,4)+0.168(5,2,2)$ \\
\hline 234 & & 10746.09 & 2.20 & 0.04 & $0.999(5,6,0)$ \\
\hline 235 & 10761.97 & 10754.37 & -0.99 & 0.03 & $0.996(2,1,5)$ \\
\hline 236 & & 10849.89 & -5.27 & 2.34 & $0.981(1,13,0)+0.116(1,9,2)-0.113[0,1,1]$ \\
\hline 237 & 10869.80 & 10854.53 & -14.28 & 3.57 & $0.976(1,11,1)+0.124[0,1,0]+0.102[0,2,0]$ \\
\hline 238 & & 10876.61 & 5.09 & 0.00 & $0.998(8,1,0)$ \\
\hline 239 & & 10879.00 & 9.70 & 5.46 & $0.949(1,9,2)-0.199[0,1,1]-0.148(1,13,0)$ \\
\hline 240 & 10901.50 & 10889.80 & -2.69 & 0.88 & $0.752(1,7,3)-0.627(4,6,1)-0.118(4,2,3)-0.110(1,11,1)$ \\
\hline 241 & & 10890.98 & 2.76 & 0.12 & $0.968(4,4,2)-0.241(1,5,4)$ \\
\hline 242 & 10907.11 & 10897.58 & 4.08 & 0.04 & $0.971(4,2,3)+0.149(7,1,1)-0.135(1,3,5)$ \\
\hline 243 & 10916.68 & 10903.77 & -7.24 & 1.82 & $0.766(4,6,1)+0.624(1,7,3)$ \\
\hline 244 & & 10912.02 & -5.64 & 0.00 & $0.996(4,0,4)$ \\
\hline 245 & & 10928.51 & -8.35 & 0.67 & $0.862(4,8,0)+0.483(1,5,4)+0.117(4,4,2)$ \\
\hline 246 & & 10944.52 & 5.85 & 0.41 & $0.831(1,5,4)-0.498(4,8,0)+0.212(4,4,2)$ \\
\hline 247 & 10964.46 & 10952.77 & -4.54 & 0.00 & $0.987(7,1,1)-0.141(4,2,3)$ \\
\hline 248 & 10990.71 & 10981.98 & 0.62 & 0.29 & $0.983(1,3,5)+0.147(4,2,3)$ \\
\hline 4 & 11004.86 & 10999.42 & 1.38 & 82.75 & $0.907[1,0,0]-0.331(0,13,1)+0.162(3,8,1)$ \\
\hline 249 & & 11041.63 & 3.55 & 0.06 & $0.994(1,1,6)$ \\
\hline 250 & & 11063.35 & 2.72 & 0.00 & $0.999(7,3,0)$ \\
\hline 251 & & 11070.28 & -2.84 & 25.81 & $\begin{array}{l}0.636(0,11,2)+0.502(0,15,0)-0.493[0,1,1]-0.167(1,9,2)+ \\
0.113(0,7,4)\end{array}$ \\
\hline 252 & $\mathrm{~b}$ & 11095.50 & -14.23 & 13.89 & $\begin{array}{l}0.731(0,13,1)+0.514(3,8,1)+0.253[0,2,0]+0.212(0,9,3)+ \\
0.192[1,0,0]+0.158[0,0,2]\end{array}$ \\
\hline 253 & & 11096.92 & 4.91 & 4.83 & $0.856(0,15,0)-0.426(0,11,2)+0.209[0,1,1]-0.117(3,6,2)$ \\
\hline 254 & 11115.74 & 11102.25 & -15.83 & 9.83 & $\begin{array}{l}0.745(3,8,1)-0.533(0,9,3)-0.259[1,0,0]-0.233(0,13,1) \\
-0.116[0,0,2]\end{array}$ \\
\hline 255 & & 11116.84 & 5.04 & 0.60 & $0.958(3,6,2)-0.181(0,7,4)-0.163(0,11,2)+0.103(3,10,0)$ \\
\hline 256 & 11133.12 & 11123.02 & -2.67 & 0.35 & $0.982(3,4,3)-0.138(0,5,5)$ \\
\hline 257 & & 11126.63 & 10.08 & 0.00 & $0.996(6,1,2)$ \\
\hline 258 & & 11137.79 & 4.20 & 2.21 & $0.963(3,10,0)-0.153(0,11,2)-0.137[0,1,1]-0.119(3,6,2)$ \\
\hline 259 & 11156.13 & 11142.35 & 9.75 & 10.02 & $\begin{array}{l}0.712(0,9,3)-0.500(0,13,1)+0.342(3,8,1)-0.195[1,0,0]+ \\
0.184[0,2,0]+0.120[0,0,2]\end{array}$ \\
\hline 260 & & 11156.13 & 3.70 & 0.06 & $0.991(3,2,4)-0.109(0,3,6)$ \\
\hline 261 & 11180.48 & 11167.17 & -2.95 & 0.02 & $0.999(6,3,1)$ \\
\hline 262 & & 11170.35 & 1.86 & 3.03 & $\begin{array}{l}0.906(0,7,4)-0.308(0,11,2)-0.143(3,10,0)- \\
0.137[0,1,1]+0.131(3,6,2)\end{array}$ \\
\hline 263 & 11193.62 & 11186.40 & -7.36 & 0.00 & $0.996(3,0,5)$ \\
\hline 264 & 11216.76 & 11206.02 & -3.47 & 22.33 & $0.862(0,5,5)-0.439[0,2,0]+0.147[0,0,2]+0.129(3,4,3)$ \\
\hline 5 & & 11220.93 & -0.45 & 46.44 & $\begin{array}{l}0.673[0,1,1]+0.480(0,11,2)+0.308(0,7,4)-0.238(2,8,2)+ \\
0.191(3,10,0)-0.151(2,12,0)+0.141(3,6,2)+0.134(1,9,2)- \\
0.127(1,10,2)\end{array}$ \\
\hline 6 & 11218.60 & 11210.65 & 5.99 & 67.44 & $\begin{array}{l}0.799[0,2,0]+0.456(0,5,5)-0.239(0,9,3)-0.176[0,0,2]- \\
0.143(3,8,1)+0.110(2,10,1)\end{array}$ \\
\hline 265 & & 11251.43 & 0.89 & 0.02 & $1.000(6,5,0)$ \\
\hline 266 & & 11259.64 & 3.18 & 0.53 & $0.988(0,3,6)+0.109(3,2,4)$ \\
\hline 7 & 11289.51 & 11283.15 & 1.28 & 73.00 & $\begin{array}{l}0.848[0,0,2]+0.316(2,10,1)-0.257(0,9,3)+0.165(2,6,3)- \\
0.113(0,13,1)\end{array}$ \\
\hline
\end{tabular}


TABLE II. (Continued.)

\begin{tabular}{|c|c|c|c|c|c|}
\hline No. & $\begin{array}{c}\text { Energy } \\
\left(\mathrm{cm}^{-1}\right) \\
K=N=1\end{array}$ & $\begin{array}{c}\text { Energy } \\
\left(\mathrm{cm}^{-1}\right) \\
K=N=0\end{array}$ & $\begin{array}{c}\text { obs. }- \text { calc. } \\
\text { error } \\
\left(\mathrm{cm}^{-1}\right)\end{array}$ & $\% A^{2} B_{2}$ & Eigenvector decomposition \\
\hline 267 & 11330.12 & 11323.62 & 2.13 & 0.11 & $0.994(0,1,7)$ \\
\hline 268 & 11343.61 & 11333.99 & 4.18 & 0.00 & $0.976(5,1,3)+0.199(8,0,1)$ \\
\hline 269 & & 11346.62 & 1.68 & 7.35 & $0.583(2,8,2)-0.562(5,3,2)-0.559(2,12,0)+0.130(2,4,4)$ \\
\hline 270 & 11363.15 & 11348.40 & -1.69 & 6.57 & $0.872(2,10,1)-0.393(2,6,3)-0.197[0,0,2]-0.128[0,2,0]$ \\
\hline 271 & & 11354.63 & 9.79 & 0.47 & $0.801(5,3,2)+0.413(2,8,2)-0.398(2,12,0)-0.138(2,4,4)$ \\
\hline 272 & & 11363.15 & 3.86 & 0.00 & $0.997(9,0,0)$ \\
\hline 273 & 11392.43 & 11381.46 & 21.95 & 8.50 & $\begin{array}{l}0.856(2,6,3)+0.318(2,10,1)-0.264(5,5,1)-0.250[0,0,2] \\
-0.109[0,2,0]\end{array}$ \\
\hline 274 & & 11376.48 & 18.41 & 0.97 & $0.707(2,12,0)+0.632(2,8,2)+0.249[0,1,1]+0.100(0,11,2)$ \\
\hline 275 & $\mathrm{~b}$ & 11379.42 & -5.62 & 0.60 & $0.961(5,5,1)+0.262(2,6,3)$ \\
\hline 276 & & 11381.20 & -6.30 & 0.41 & $0.977(2,4,4)+0.187(5,3,2)$ \\
\hline 277 & 11410.73 & 11400.23 & -12.14 & 0.00 & $0.976(8,0,1)-0.191(5,1,3)+0.106(2,2,5)$ \\
\hline 278 & 11430.99 & 11422.84 & -3.51 & 0.10 & $0.988(2,2,5)$ \\
\hline 279 & & 11440.67 & -2.05 & 0.15 & $0.998(5,7,0)$ \\
\hline 280 & & 11477.76 & -2.35 & 0.00 & $0.997(2,0,6)$ \\
\hline 281 & & 11547.93 & 2.07 & 0.00 & $0.970(7,0,2)+0.242(4,1,4)$ \\
\hline 282 & $\mathrm{~b}$ & 11553.37 & -13.81 & 0.13 & $0.969(4,3,3)+0.176(7,2,1)-0.152(1,4,5)$ \\
\hline 283 & & 11557.04 & 1.70 & 0.00 & $0.997(8,2,0)$ \\
\hline 284 & & 11570.90 & 0.98 & 0.50 & $0.957(4,5,2)-0.257(1,6,4)$ \\
\hline 285 & & 11576.34 & 9.45 & 2.16 & $0.917(1,14,0)-0.351(1,10,2)-0.100[0,2,1]$ \\
\hline 286 & & 11593.63 & 3.85 & 7.02 & $\begin{array}{l}0.867(1,10,2)+0.362(1,14,0)+0.186[0,2,1]-0.185(4,9,0) \\
+0.167[0,1,1]\end{array}$ \\
\hline 287 & 11580.82 & 11568.20 & -13.09 & 3.26 & $0.741(1,8,3)-0.602(4,7,1)-0.210(1,12,1)-0.115[0,1,2]$ \\
\hline 288 & 11591.20 & 11574.30 & -1.35 & 4.89 & $0.943(1,12,1)-0.233(4,7,1)+0.127[1,1,0]-0.104[0,0,2]$ \\
\hline 289 & & 11601.89 & 15.66 & 0.01 & $0.963(4,1,4)-0.242(7,0,2)$ \\
\hline 290 & $\mathrm{~b}$ & 11604.43 & 4.18 & 6.86 & $\begin{array}{l}0.731(4,7,1)+0.599(1,8,3)-0.161[1,1,0]+0.153(1,12,1) \\
-0.146[0,1,2]-0.101[0,0,2]\end{array}$ \\
\hline 291 & & 11619.38 & -0.86 & 2.26 & $0.941(1,6,4)+0.245(4,5,2)$ \\
\hline 292 & 11638.61 & 11624.79 & -9.21 & 0.01 & $0.981(7,2,1)-0.161(4,3,3)+0.104(1,4,5)$ \\
\hline 293 & & 11635.86 & -1.77 & 2.75 & $0.961(4,9,0)+0.171(1,10,2)+0.149[1,0,1]$ \\
\hline 294 & 11664.04 & 11654.32 & -3.21 & 0.63 & $0.973(1,4,5)+0.167(4,3,3)$ \\
\hline 8 & & 11694.41 & 0.97 & 71.40 & $\begin{array}{l}0.841[1,1,0]+0.319(0,14,1)-0.274(3,9,1)+0.197(4,7,1) \\
+0.126(0,10,3)\end{array}$ \\
\hline 295 & & 11704.48 & -0.20 & 0.21 & $0.990(1,2,6)+0.101(4,1,4)$ \\
\hline 9 & & 11720.41 & a & 60.6 & $\begin{array}{l}0.771[1,0,1]-0.349(0,12,2)-0.292(0,16,0)+0.243(3,7,2) \\
+0.180(3,11,0)-0.180(4,9,0)\end{array}$ \\
\hline 296 & & 11742.84 & -6.09 & 0.01 & $0.999(7,4,0)$ \\
\hline 297 & 11768.58 & 11759.14 & 8.15 & 0.00 & $0.999(6,0,3)$ \\
\hline 298 & 11783.85 & 11776.96 & 7.96 & 0.01 & $0.997(1,0,7)$ \\
\hline
\end{tabular}

${ }^{\mathrm{a}}$ Calculated frequency.

${ }^{\mathrm{b}} K=N=0$ limit deduced from $K=0, N=1,3,5, \ldots$ LIF, ICLAS or CRDS experimental energies.

shifts discussed in the paragraph above. Interestingly, one however also observes a certain number of interactions between two states of the ground electronic surface, which are coupled by non-negligible $\left\langle\psi_{m}^{g}|\mathbf{H}| \psi_{n}^{g}\right\rangle$ matrix elements [see Eq. (3.6)]. Nonetheless, due the much smaller value for $\lambda$ $\left(332 \mathrm{~cm}^{-1}\right.$ against more than $2400 \mathrm{~cm}^{-1}$ ), the mixing coefficients reported in the two last columns of Table II remain much smaller than those obtained by Leonardi and Petrongolo. ${ }^{11}$

\section{CONCLUSION}

We have derived an efficient method for adjusting the parameters of an effective Hamiltonian against experimental data. This model accurately reproduces the observed frequencies up to a few thousands of $\mathrm{cm}^{-1}$ above the conical intersection. Continuation of this work is expected in two directions. First, we plan to use the effective Hamiltonian derived in this article to reproduce the absorption spectra, which have been recorded by the ICLAS and FTS techniques in the range $11200-16150 \mathrm{~cm}^{-1}$.,17 While the goal would, of course, no longer be to calculate all transition energies with an error of the order of a few $\mathrm{cm}^{-1}$, a visual or statistical comparison of the observed and calculated spectra should be able to indicate up to what energies such a model remains valid, and eventually what corrections are needed. Moreover, we plan to analyze the classical phase space of the vibronically coupled system along the lines proposed in Refs. 18 and 19. The underlying idea is to use the classical periodic orbits of the system to study the nonadiabatic quantum dynamics, as was done recently for several triatomic molecules in the context of the Born-Oppenheimer approximation (see, for example, Refs. 20 and 21, and references therein). 


\section{APPENDIX: CALCULATION \\ OF THE $\left\langle v_{1}^{\prime}, v_{2}^{\prime}, v_{3}^{\prime}\left|q_{3}^{n}\right| v_{1}, v_{2}, v_{3}\right\rangle$ INTEGRALS}

Since, for symmetry reasons, the matrix $\mathbf{A}=\left\{A_{j k}\right\}$ is block diagonal [cf. Eq. (2.6)], the integrals to compute split in the more manageable form,

$$
\left\langle v_{1}^{\prime}, v_{2}^{\prime}, v_{3}^{\prime}\left|q_{3}^{n}\right| v_{1}, v_{2}, v_{3}\right\rangle=\left\langle v_{1}^{\prime}, v_{2}^{\prime} \mid v_{1}, v_{2}\right\rangle\left\langle v_{3}^{\prime}\left|q_{3}^{n}\right| v_{3}\right\rangle .
$$

After some straightforward algebra, the second integral in the right-hand side of Eq. (A1) is rewritten as

$$
\begin{aligned}
\left\langle v_{3}^{\prime}\left|q_{3}^{n}\right| v_{3}\right\rangle= & C_{3}\left(n, v_{3}, v_{3}^{\prime}\right) \\
& \times \int_{-\infty}^{+\infty} P_{3}\left(n, v_{3}, v_{3}^{\prime} \mid x_{3}\right) e^{-x_{3}^{2}} d x_{3},
\end{aligned}
$$

where

$$
\begin{aligned}
& \alpha_{3}=\sqrt{\frac{2}{1+A_{33}^{2}}}, \\
& C_{3}\left(n, v_{3}, v_{3}^{\prime}\right)=\alpha_{3}^{1+n} \sqrt{\frac{A_{33}}{\pi v_{3} ! v_{3}^{\prime} ! 2^{v_{3}+v_{3}^{\prime}}}} \\
& q_{3}=\alpha_{3} x_{3}, \\
& P_{3}\left(n, v_{3}, v_{3}^{\prime} \mid x_{3}\right)=x_{3}^{n} H_{v_{3}}\left(\alpha_{3} x_{3}\right) H_{v_{3}^{\prime}}\left(A_{33} \alpha_{3} x_{3}\right),
\end{aligned}
$$

and the $H_{m}$ 's are the Hermite polynomials of order $m$. The polynomial $P_{3}\left(n, v_{3}, v_{3}^{\prime} \mid x_{3}\right)$ is next projected on the orthogonal basis of the Hermite polynomials, leading to

$$
P_{3}\left(n, v_{3}, v_{3}^{\prime} \mid x_{3}\right)=\sum_{k=0}^{n+v_{3}+v_{3}^{\prime}} a_{3}\left(n, v_{3}, v_{3}^{\prime} \mid k\right) H_{k}\left(x_{3}\right) .
$$

Remembering that the term of $H_{k}(x)$ with highest order is $2^{k} x^{k}$, the real coefficients $a_{3}\left(n, v_{3}, v_{3}^{\prime} \mid k\right)$ are easily obtained downwards, starting with $a_{3}\left(n, v_{3}, v_{3}^{\prime} \mid n+v_{3}+v_{3}^{\prime}\right)$ and ending with $a_{3}\left(n, v_{3}, v_{3}^{\prime} \mid 0\right)$. The second integral in the righthand side of Eq. (A1) is then just

$$
\left\langle v_{3}^{\prime}\left|q_{3}^{n}\right| v_{3}\right\rangle=\sqrt{\pi} C_{3}\left(n, v_{3}, v_{3}^{\prime}\right) a_{3}\left(n, v_{3}, v_{3}^{\prime} \mid 0\right) .
$$

Calculation of the first integral in the right-hand side of Eq. (A1) is somewhat more tedious, but this can again be performed without ever integrating a function numerically, which is of fundamental importance for the sake of accuracy. Proceeding along the same lines as in Refs. 4 and 22, one first obtains, by inverting Eq. (2.6), the matrices $\mathbf{T}$ and $\mathbf{D}$, such that

$$
\left(\begin{array}{l}
q_{1} \\
q_{2}
\end{array}\right)=\mathbf{T}\left(\begin{array}{l}
q_{1}^{\prime} \\
q_{2}^{\prime}
\end{array}\right)+\mathbf{D} .
$$

One then calculates the orthogonal matrix $\mathbf{W}$ and the diagonal matrix $\mathbf{G}$ of, respectively, the eigenvectors and eigenvalues of ${ }^{t} \mathbf{T} \mathbf{T}$,

$$
{ }^{t} \mathbf{T} \mathbf{T} \mathbf{W}=\mathbf{W} \mathbf{G} .
$$

The matrix $\mathbf{U}$ and the vectors $\mathbf{d}$ and $\mathbf{s}$ are defined from $\mathbf{W}$ and $\mathbf{G}$ according to

$$
\mathbf{U}=\mathbf{T} \mathbf{W},
$$

$$
\begin{aligned}
& \mathbf{d}=\mathbf{G}^{1 / 2} \mathbf{U}^{-1} \mathbf{D}, \\
& \mathbf{s}=\left(\begin{array}{l}
s_{1} \\
s_{2}
\end{array}\right)=\mathbf{W}^{-1}\left(\begin{array}{l}
q_{1}^{\prime} \\
q_{2}
\end{array}\right) .
\end{aligned}
$$

One finally notes

$$
\begin{aligned}
& \alpha_{j}=\sqrt{\frac{2}{1+G_{j j}}}, \\
& \beta_{j}=\frac{d_{j} \sqrt{G_{j j}}}{1+G_{j j}}, \\
& \gamma_{j}=\frac{d_{j}^{2}}{2\left(1+G_{j j}\right)}, \\
& s_{j}=\alpha_{j} x_{j}-\beta_{j}
\end{aligned}
$$

$(j=1,2)$. After some straightforward algebra, whose first steps are sketched in Refs. 4 and 22, the first integral in the right-hand side of Eq. (A1) is recast in the form,

$$
\begin{aligned}
\left\langle v_{1}^{\prime}, v_{2}^{\prime} \mid v_{1}, v_{2}\right\rangle= & C\left(v_{1}, v_{2}, v_{1}^{\prime}, v_{2}^{\prime}\right) \\
& \times \sum_{i_{1}=0}^{v_{1}} \sum_{i_{2}=0}^{v_{2}} \sum_{i_{1}^{\prime}=0}^{v_{1}^{\prime}} \sum_{i_{2}^{\prime}=0}^{v_{2}^{\prime}}\left(\begin{array}{c}
v_{1} \\
i_{1}
\end{array}\right)\left(\begin{array}{c}
v_{2} \\
i_{2}
\end{array}\right)\left(\begin{array}{c}
v_{1}^{\prime} \\
i_{1}^{\prime}
\end{array}\right)\left(\begin{array}{c}
v_{2}^{\prime} \\
i_{2}^{\prime}
\end{array}\right) \\
& \times \int_{-\infty}^{+\infty} P_{1}\left(i_{1}, i_{2}, i_{1}^{\prime}, i_{2}^{\prime} \mid x_{1}\right) e^{-x_{1}^{2}} d x_{1} \\
& \times \int_{-\infty}^{+\infty} P_{2}\left(v_{1}-i_{1}, v_{2}-i_{2}, v_{1}^{\prime}-i_{1}^{\prime}, v_{2}^{\prime}\right. \\
& \left.-i_{2}^{\prime} \mid x_{2}\right) e^{-x_{2}^{2}} d x_{2},
\end{aligned}
$$

where

$$
\begin{aligned}
& C\left(v_{1}, v_{2}, v_{1}^{\prime}, v_{2}^{\prime}\right) \\
& =\frac{\alpha_{1} \alpha_{2} e^{-\gamma_{1}-\gamma_{2}} \operatorname{det}(\mathbf{W})}{\pi 2^{v_{1}+v_{2}+v_{1}^{\prime}+v_{2}^{\prime}}} \sqrt{\frac{\operatorname{det}(\mathbf{T})}{v_{1} ! v_{2} ! v_{1}^{\prime} ! v_{2}^{\prime} !}}, \\
& \begin{aligned}
P_{1}\left(i_{1}, i_{2}, i_{1}^{\prime}, i_{2}^{\prime} \mid x_{1}\right)= & H_{i_{1}}\left(\sqrt{2}\left(U_{11} s_{1}+D_{1}\right)\right) H_{i_{2}}\left(\sqrt{2} U_{21} s_{1}\right) \\
& \times H_{i_{1}^{\prime}}\left(\sqrt{2} W_{11} s_{1}\right) H_{i_{2}^{\prime}}\left(\sqrt{2} W_{21} s_{1}\right),
\end{aligned}
\end{aligned}
$$

$$
\begin{aligned}
P_{2}\left(i_{1}, i_{2}, i_{1}^{\prime}, i_{2}^{\prime} \mid x_{2}\right)= & H_{i_{1}}\left(\sqrt{2} U_{12} s_{2}\right) H_{i_{2}}\left(\sqrt{2}\left(U_{22} s_{2}+D_{2}\right)\right) \\
& \times H_{i_{1}^{\prime}}\left(\sqrt{2} W_{12} s_{2}\right) H_{i_{2}^{\prime}}\left(\sqrt{2} W_{22} s_{2}\right),
\end{aligned}
$$

and the $s_{j}$ 's must be replaced with their expressions in terms of the $x_{j}$ 's in Eq. (A9). As for the integral dealing with mode 3 , each polynomial $P_{j}\left(i_{1}, i_{2}, i_{1}^{\prime}, i_{2}^{\prime} \mid x_{j}\right)(j=1,2)$ is next projected on the orthogonal basis of the Hermite polynomials, according to

$$
P_{j}\left(i_{1}, i_{2}, i_{1}^{\prime}, i_{2}^{\prime} \mid x_{j}\right)=\sum_{k=0}^{i_{1}+i_{2}+i_{1}^{\prime}+i_{2}^{\prime}} a_{j}\left(i_{1}, i_{2}, i_{1}^{\prime}, i_{2}^{\prime} \mid k\right) H_{k}\left(x_{j}\right),
$$

so that the corresponding integrals are equal to

$$
\int_{-\infty}^{+\infty} P_{j}\left(i_{1}, i_{2}, i_{1}^{\prime}, i_{2}^{\prime} \mid x_{j}\right) e^{-x_{j}^{2}} d x_{j}=\sqrt{\pi} a_{j}\left(i_{1}, i_{2}, i_{1}^{\prime}, i_{2}^{\prime} \mid 0\right)
$$


$(j=1,2)$. It is stressed, that the number of accuracy digits, which are lost when computing the real coefficients $a_{j}$ and the quadruple sum in Eq. (A10), is sometimes larger than 50 for the $\left\langle v_{1}^{\prime}, v_{2}^{\prime}, v_{3}^{\prime}\left|q_{3}^{n}\right| v_{1}, v_{2}, v_{3}\right\rangle$ integrals used in this work. Therefore, one has to evaluate these integrals with packages, which allow for arbitrarily large numbers of working digits, and to check that the number of significant digits at the end of the calculations is still large enough.

${ }^{1}$ A. Delon and R. Jost, J. Chem. Phys. 95, 5686 (1991).

${ }^{2}$ R. Georges, A. Delon, F. Bylicki, R. Jost, A. Campargue, A. Charvat, M. Chenevier, and F. Stoeckel, Chem. Phys. 190, 207 (1995).

${ }^{3}$ B. Kirmse, A. Delon, and R. Jost, J. Chem. Phys. 108, 6638 (1998).

${ }^{4}$ A. Delon, R. Jost, and M. Jacon, J. Chem. Phys. 114, 331 (2001).

${ }^{5}$ A. Delon, R. Jost, and M. Lombardi, J. Chem. Phys. 95, 5701 (1991).

${ }^{6}$ E. Haller, H. Köppel, and L. S. Cederbaum, J. Mol. Spectrosc. 111, 377 (1985).

${ }^{7}$ S. Mahapatra, H. Köppel, and L. S. Cederbaum, J. Chem. Phys. 110, 5691 (1999).
${ }^{8}$ S. Mahapatra, H. Köppel, L. S. Cederbaum, P. Stampfuss, and W. Wenzel, Chem. Phys. 259, 211 (2000).

${ }^{9}$ R. F. Salzgeber, V. A. Mandelshtam, Ch. Schlier, and H. S. Taylor, J. Chem. Phys. 110, 3756 (1999).

${ }^{10}$ E. Leonardi, C. Petrongolo, G. Hirsch, and R. J. Buenker, J. Chem. Phys. 105, 9051 (1996)

${ }^{11}$ E. Leonardi and C. Petrongolo, J. Chem. Phys. 106, 10066 (1997).

${ }^{12}$ R. Brandi, F. Santoro, and C. Petrongolo, Chem. Phys. 225, 55 (1997).

${ }^{13}$ F. Santoro and C. Petrongolo, J. Chem. Phys. 110, 4419 (1999).

${ }^{14}$ R. Jost, M. Joyeux, and M. Jacon, Chem. Phys. 283, 17 (2002).

${ }^{15}$ D. Sugny and M. Joyeux, Chem. Phys. Lett. 337, 319 (2001).

${ }^{16}$ M. Joyeux, D. Sugny, and M. Lombardi, Chem. Phys. Lett. 352, 99 (2002).

${ }^{17}$ J. Orphal, S. Dreher, S. Voigt, J. P. Burrows, R. Jost, and A. Delon, J. Chem. Phys. 109, 10217 (1998).

${ }^{18}$ S. Dilthey and G. Stock, Phys. Rev. Lett. 87, 140404 (2001).

${ }^{19}$ S. Dilthey, B. Mehlig, and G. Stock, J. Chem. Phys. 116, 69 (2002).

${ }^{20}$ H. Ishikawa, R. W. Field, S. C. Farantos, M. Joyeux, J. Koput, C. Beck, and R. Schinke, Annu. Rev. Phys. Chem. 50, 443 (1999).

${ }^{21}$ M. Joyeux, S. C. Farantos, and R. Schinke, J. Phys. Chem. A 106, 5407 (2002).

${ }^{22}$ T. R. Faulkner and F. S. Richardson, J. Chem. Phys. 70, 1201 (1979). 